\title{
ESTIMATION OF DERIVATIVES FOR REGULAR POSITIVE REAL PART FUNCTIONS
}

\author{
WENFA YUAN ${ }^{1}$, DONGLI CHEN ${ }^{1}$ AND HUIGUANG KANG ${ }^{2}$
}

\begin{abstract}
In this paper, we mainly discuss the problem of estimating the $n$th derivative of regular positive real part functions: $g(z)=c_{0}+c_{1} z+\cdots+c_{n} z^{n}+\cdots$, which is regular in $|z|<1$ and $\operatorname{Re} g(z)>0$. With the principle of inductive method and the characters of regular positive real part functions, the estimation of the $n$th derivative for the function $g(z)$ is presented. The derivative estimation for positive functions with real part has been solved completely.
\end{abstract}

\section{Introduction}

Consider the following family of the function:

$$
B=\left\{\varphi(z) \mid \varphi(z)=c+c_{1} z+\cdots+c_{n} z^{n}+\cdots, \text { and }|\varphi(z)|<1\right\} .
$$

It is well known that

$$
\left|\varphi^{\prime}(z)\right| \leq \frac{1-|\varphi(z)|^{2}}{1-|z|^{2}}
$$

Also Pan and Liao ([3]) has obtained the derivative estimation for the family $B$ of the functions, i.e.. Let $\varphi(z)=c_{0}+c_{1} z+\cdots+c_{n} z^{n}+\cdots$ be regular in $|z|<1$ and $\mid \varphi(z)<1$, then

$$
\left|\varphi^{\prime \prime}(z)\right| \leq \frac{2(1+|z|)}{\left(1-|z|^{2}\right)^{2}}\left(1-|\varphi(z)|^{2}\right) .
$$

And at the same time they gave the following estimation of first and second derivatives of regular function with positive real part:

$$
\begin{aligned}
\left|g^{\prime}(z)\right| & \leq \frac{2 \operatorname{Re} g(z)}{1-|z|^{2}}, \\
\left|g^{\prime \prime}(z)\right| & =\frac{4(1+|z|)}{\left(1-|z|^{2}\right)^{2}} \operatorname{Re} g(z) .
\end{aligned}
$$

$\overline{\text { Received and revised December 16, } 2003 .}$

2000 Mathematics Subject Classification. 30C10.

Key words and phrases. Regular positively real part function, regular function, derivatives, estimation.

Supported by the Special Science Foundation of the Educational Committee of Shaanxi Province (03JK066).

Supported by the Science Foundation of Xi'an University of Architecture \& Technology (02BR04). 
We have to point out that there is an error in the Pan and Liao's ([3]) 3rd derivative estimation and Yuan ([5]) got the universal formula for $\left(n^{\text {th }}\right)$ derivative of regularly bounded functions. This paper is to investigate the derivative estimation for regular function with positive real part further to obtain its estimation, and consequently the special estimation for derivative is generalized to usual one.

\section{Main Result}

Theorem. Let $g(z)=a_{0}+a_{1} z+\cdots+a_{n} z^{n}+\cdots$ be regular in $|z|<1$ and $\operatorname{Re} g(z)>0$, then we have

$$
\left|g^{(n)}(z)\right| \leq \frac{2 n ! \sum_{m=0}^{n-1} I(n, m)|z|^{m}}{\left(1-|z|^{2}\right)^{n}} \operatorname{Re} g(z),
$$

where

$$
\begin{aligned}
I(n, 0)=1, I(n, 1)=n-1, I(n, m)=\sum_{k=1}^{m}\left(\begin{array}{c}
n-1 \\
n-k-1
\end{array}\right) I(n-k, m-k), \\
m \leq n-1, m=1,2, \ldots, n-1 .
\end{aligned}
$$

Some Lemma was given before we complete the proof of Theorem.

Lemma 1.([1]) Let $\varphi(z)=c_{0}+c_{1} z+c_{2} z^{2}+\cdots+c_{n} z^{n}+\cdots$ be regualr in $|z|<1$ and $|\varphi(z)|<1$, then we have

$$
\varphi\left(\frac{s+z}{1+\bar{z} s}\right)=\sum_{n=0}^{\infty} g_{n}(z) s^{n}
$$

where $|s|<1, g_{0}=\varphi(z)$,

$$
g_{n}(z)=\sum_{j=0}^{n-1} \frac{(-1)^{j}\left(\begin{array}{c}
n-1 \\
j
\end{array}\right)}{(n-j) !} \bar{z}^{j}\left(1-|z|^{2}\right)^{n-j} \varphi^{(n-j)}(z), \quad(n \geq 1) .
$$

Lemma 2. Let $\varphi(z)=c_{0}+c_{1} z+c_{2} z^{2}+\cdots+c_{n} z^{n}+\cdots$ be regualr in $|z|<1$ and $|\varphi(z)|<1$, then

$$
\left|\frac{\varphi^{(n)}(z)\left(1-|z|^{2}\right)^{n}}{n !}\right| \leq\left(1-|\varphi(z)|^{2}\right)+\sum_{v=1}^{n-1}\left[\left(\begin{array}{c}
n-1 \\
j-1
\end{array}\right)|z|^{n-v}\left(1-|z|^{2}\right)^{v} \frac{\left|\varphi^{(v)}(z)\right|}{v !}\right] .
$$

Proof. From Lemma 1

$$
\Phi(s)=\varphi\left(\frac{s+z}{1+\bar{z} s}\right)=\sum_{n=0}^{\infty} g_{n}(z) s^{n}
$$


and its general term is given as

$$
g_{n}(z)=\sum_{j=0}^{n-1} \frac{(-1)^{j}\left(\begin{array}{c}
n-1 \\
j
\end{array}\right)}{(n-j) !} \bar{z}^{j}\left(1-|z|^{2}\right)^{n-j} \varphi^{(n-j)}(z), \quad(n \geq 1) .
$$

Since coefficients relations of regular functions: $\left|c_{n}\right| \leq 1-\left|c_{0}\right|^{2}$, we have

$$
\left|\Phi^{(n)}(0)\right|=n !\left|g_{n}(z)\right| \leq n !\left(1-|\varphi(z)|^{2}\right) ;
$$

Combining $\left|g_{n}(z)\right| \leq 1-|\varphi(z)|^{2}$ and the above form yields

$$
\begin{aligned}
1-|\varphi(z)|^{2} & \geq\left|\sum_{j=0}^{n-1} \frac{(-1)^{j}\left(\begin{array}{c}
n-1 \\
j
\end{array}\right)}{(n-j) !} \bar{z}^{j}\left(1-|z|^{2}\right)^{n-j} \varphi^{(n-j)}(z)\right| \\
& \geq\left|\frac{\left(1-|z|^{2}\right)^{n}}{n !} \varphi^{(n)}(z)\right|-\left|\sum_{j=0}^{n-1} \frac{(-1)^{j}\left(\begin{array}{c}
n-1 \\
j
\end{array}\right)}{(n-j) !} \bar{z}^{j}\left(1-|z|^{2}\right)^{n-j} \varphi^{(n-j)}(z)\right|,
\end{aligned}
$$

Hence

$$
\left|\frac{\left(1-|z|^{2}\right)^{n}}{n !} \varphi^{(n)}(z)\right| \leq\left(1-|\varphi(z)|^{2}\right)+\sum_{j=0}^{n-1} \frac{\left(\begin{array}{c}
n-1 \\
j
\end{array}\right)}{(n-j) !}|z|^{j}\left(1-|z|^{2}\right)^{n-j}\left|\varphi^{(n-j)}(z)\right|,
$$

and let $v=n-j$ we complete the proof.

Lemma 3. If $g(z)=c_{0}+c_{1} z+c_{2} z^{2}+\cdots+c_{n} z^{n}+\cdots$ be regualr in $|z|<1$ and $\operatorname{Re} g(z)>0$, then

$$
\left|\frac{g^{(n)}(z)\left(1-|z|^{2}\right)^{n}}{n !}\right| \leq 2 \operatorname{Re} g(z)+\sum_{v=1}^{n-1}\left[\left(\begin{array}{c}
n-1 \\
v-1
\end{array}\right)|z|^{n-v}\left(1-|z|^{2}\right)^{v} \frac{\left|g^{(v)}(z)\right|}{v !}\right] .
$$

Proof. Consider the function

$$
G(s)=g\left(\frac{z+s}{1+\bar{z} s}\right), \quad(|s|<1) .
$$

Note that coefficients of expansion for positively real part function

$$
\left|a_{n}\right| \leq 2 \operatorname{Re}\left\{a_{0}\right\},
$$

we reach

$$
\left|G^{(n)}(0)\right| \leq 2 n ! \operatorname{Re} g(z)
$$

From Lemma 1, we get

$$
G^{(n)}(0)=\sum_{j=0}^{n-1} \frac{(-1)^{j}\left(\begin{array}{c}
n-1 \\
j
\end{array}\right)}{(n-j) !} \bar{z}^{j}\left(1-|z|^{2}\right)^{n-j} g^{(n-j)}(z), \quad(n \geq 1) .
$$


So

$$
\left|\sum_{j=0}^{n-1} \frac{(-1)^{j}\left(\begin{array}{c}
n-1 \\
j
\end{array}\right)}{(n-j) !} \bar{z}^{j}\left(1-|z|^{2}\right)^{n-j} g^{(n-j)}(z)\right| \leq 2 n ! \operatorname{Re} g(z), \quad(n \geq 1) .
$$

By Lemma 2, triangle inequality and let $v=n-j$, then we obtain the following inequality

$$
\left|\frac{g^{(n)}(z)\left(1-|z|^{2}\right)^{n}}{n !}\right| \leq 2 \operatorname{Re} g(z)+\sum_{v=1}^{n-1}\left[\left(\begin{array}{c}
n-1 \\
v-1
\end{array}\right)|z|^{n-v}\left(1-|z|^{2}\right)^{v} \frac{\left|g^{(v)}(z)\right|}{v !}\right]
$$

and this completes the proof.

Now let's prove the Theorem by the principle of inductive method.

Let $n=1$, we have $I(1,0)=1$, and hence

$$
\left|g^{\prime}(z)\right| \leq \frac{2 \operatorname{Re} g(z)}{1-|z|^{2}}
$$

that is, the Theorem holds.

Let $n=2$ and we get $I(2,1)=\left(\begin{array}{l}1 \\ 0\end{array}\right) I(1,0)=1, I(2,0)=1$ and

$$
\left|g^{\prime \prime}(z)\right| \leq \frac{2 \cdot 2 !(1+|z|)}{\left(1-|z|^{2}\right)^{2}} \operatorname{Re} g(z)
$$

i.e., the Theorem holds.

Suppose Theorem holds in case $n=k$, equivalently,

$$
\left|g^{(k)}(z)\right| \leq \frac{2 \cdot k ! \operatorname{Re} g(z)}{\left(1-|z|^{2}\right)^{k}} \sum_{m=0}^{k-1} I(k, m)|z|^{m}
$$

Next we prove the Theorem in case $n=k+1$. Begin with the $n^{\text {th }}$ derivative of

$$
G(s)=g\left(\frac{z+s}{1+\bar{z} s}\right)
$$

and let $s=0$ and note Lemma 3, we arrive at

$$
\left|\frac{g^{(k+1)}(z)\left(1-|z|^{2}\right)^{k+1}}{(k+1) !}\right| \leq 2 \operatorname{Re} g(z)+\sum_{v=1}^{k}\left[\left(\begin{array}{c}
k \\
v-1
\end{array}\right)|z|^{k-v+1}\left(1-|z|^{2}\right)^{v} \frac{\left|g^{(v)}(z)\right|}{v !}\right] .
$$

Now substituting (7) into (8) yields,

$$
\begin{aligned}
& \left|\frac{g^{(k+1)}(z)\left(1-|z|^{2}\right)^{k+1}}{(k+1) !}\right| \\
\leq & 2 \operatorname{Re} g(z)+\sum_{v=1}^{k} 2\left[\left(\begin{array}{c}
k \\
v-1
\end{array}\right)|z|^{k-v+1} \sum_{m=0}^{k-1} I(k, m)|z|^{m}\right] \operatorname{Re} g(z)
\end{aligned}
$$




$$
\begin{aligned}
&= 2 \operatorname{Re} g(z)\left\{1+\sum_{v=1}^{k}\left(\begin{array}{c}
k \\
v-1
\end{array}\right)|z|^{k-v+1}\left[I(k, 0)+I(k, 1)|z|+\cdots+I(k, k-1)|z|^{k-1}\right]\right\} \\
&= 2 \operatorname{Re} g(z)\left\{1+\left(\begin{array}{c}
k \\
k-1
\end{array}\right)|z|+\left[\left(\begin{array}{c}
k \\
k-1
\end{array}\right) I(k, 1)+\left(\begin{array}{c}
k \\
k-1
\end{array}\right) I(k-1, k-2)\right]|z|^{2}+\cdots\right. \\
&\left.+\left[\left(\begin{array}{c}
k \\
k-1
\end{array}\right) I(k, k-1)+\left(\begin{array}{c}
k \\
k-2
\end{array}\right) I(k-1, k-2)+\cdots+\left(\begin{array}{c}
k \\
1
\end{array}\right) I(2,1)+\left(\begin{array}{c}
k \\
0
\end{array}\right) I(1,0)\right]|z|^{k}\right\} \\
&= 2 \operatorname{Re} g(z)\left\{1+k|z|+\left[\sum_{v=1}^{2}\left(\begin{array}{c}
k \\
k-v
\end{array}\right) I(k-v+1,2-v)\right]|z|^{2}\right. \\
&+\left[\sum_{v=1}^{3}\left(\begin{array}{c}
k \\
k-v
\end{array}\right) I(k-v+1,3-v)\right]|z|^{3}+\cdots \\
&\left.+\left[\sum_{v=1}^{s}\left(\begin{array}{c}
k \\
k-v
\end{array}\right) I(k-v+1, s-v)\right]|z|^{s}+\cdots+\left[\sum_{v=1}^{k}\left(\begin{array}{c}
k \\
k-v
\end{array}\right) I(k-v+1, k-v)\right]|z|^{k}\right\} \\
&= 2 \operatorname{Re} g(z) \sum_{m=0}^{k} I(k, m)|z|^{m}, \text { i.e. } \\
&\left|g^{(k+1)}(z)\right| \leq \frac{2(k+1) ! \operatorname{Re} g(z)}{\left(1-|z|^{2}\right)^{k+1}} \sum_{m=0}^{k} I(k+1, m)|z|^{m} .
\end{aligned}
$$

Thus the proof is completed and we point out that the derivative estimation for positive functions with real part has been solved completely.

For an extremal function in the function class we will present our future presentation.

\section{References}

[1] S. Gong, A remark on Möbius transformations (I), Pure and Applied Mathematics 1(1985), $1-15$.

[2] P. Niamsup, A note on the characteristics of Möbius transformations, Journal of Mathematical Analysis and Applications 248(2000), 203-215.

[3] Y. F. Pan and X. Z. Liao, The derivates for bounded functions, Journal of Jiangxi Normal University (Natural Science) 1(1984), 21-24.

[4] D. B. Shaffer, On bounds for the derivative of analytic functions, Proceedings of the American Mathematical Society 37(1973), 517-520.

[5] W. F. Yuan, Estimation of derivatives for bounded regular functions, Chinese Journal of Mathematics 21(2001), 301-303.

[6] H. M. Srivastava and S. Owa (Editors), Univalent Functions, Fractional Calculus, and Their Applications, John Wiley and Sons, New York, 1989.

${ }^{1}$ College of Science, Xi'an University of Architecture \& Technology, Xi'an, Shaanxi 710055, P. R. China.

${ }^{2}$ Department of Mathematics, An'yang Teacher College, An'yang He'nan, 455000, P. R. China. 